\title{
Iterative Joint Channel Estimation and Multi-User Detection for Multiple-Antenna Aided OFDM Systems
}

\author{
M. Jiang, Member, IEEE, J. Akhtman, Member, IEEE, and L. Hanzo, Fellow, IEEE
}

\begin{abstract}
Multiple-Input-Multiple-Output (MIMO) Orthogonal Frequency Division Multiplexing (OFDM) systems have recently attracted substantial research interest. However, compared to Single-Input-Single-Output (SISO) systems, channel estimation in the MIMO scenario becomes more challenging, owing to the increased number of independent transmitterreceiver links to be estimated. In the context of the Bell LAyered Space-Time architecture (BLAST) or Space Division Multiple Access (SDMA) multi-user MIMO OFDM systems, none of the known channel estimation techniques allows the number of users to be higher than the number of receiver antennas, which is often referred to as a "rank-deficient" scenario, owing to the constraint imposed by the rank of the MIMO channel matrix. Against this background, in this paper we propose a new Genetic Algorithm (GA) assisted iterative Joint Channel Estimation and Multi-User Detection (GA-JCEMUD) approach for multi-user MIMO SDMA-OFDM systems, which provides an effective solution to the multi-user MIMO channel estimation problem in the above-mentioned rank-deficient scenario. Furthermore, the GAs invoked in the data detection literature can only provide a hard-decision output for the Forward Error Correction (FEC) or channel decoder, which inevitably limits the system's achievable performance. By contrast, our proposed GA is capable of providing "soft" outputs and hence it becomes capable of achieving an improved performance with the aid of FEC decoders. A range of simulation results are provided to demonstrate the superiority of the proposed scheme.
\end{abstract}

Index Terms-Channel estimation, genetic algorithm, multiple-input-multiple-output, multi-user detection, orthogonal frequency division multiplexing, space division multiple access.

\section{INTRODUCTION}

$\mathbf{M}$ ULTIPLE-Input-Multiple-Output (MIMO) Orthogonal Frequency Division Multiplexing (OFDM) systems have recently attracted substantial research interest [1]-[3]. Specifically, intensive research efforts have been invested both in Bell Labs Layered Space-Time architecture (BLAST) and in Space Division Multiple Access (SDMA) based MIMO OFDM [4]. In SDMA-OFDM systems the transmitted signals

Manuscript received January 25, 2006; revised October 19, 2006; accepted June 14, 2007. The associate editor coordinating the review of this paper and approving it for publication was X. Wang. The work reported in this paper has formed part of the Wireless Enablers 2.2 of the Core 3 Research Programme of the Virtual Centre of Excellence in Mobile and Personal Communications, Mobile VCE, www.mobilevce.com, whose funding support, including that of EPSRC, is gratefully acknowledged. Fully detailed technical reports on this research are available to Industrial Members of Mobile VCE.

M. Jiang was with the School of ECS, University of Southampton, Southampton, UK. He is now with Samsung Electronics Research Institute, Staines TW18 4QE, UK (email: ming.jiang@samsung.com).

J. Akhtman and L. Hanzo are with the School of ECS, University of Southampton, Southampton, UK (email: \{ya, lh $\} @$ ecs.soton.ac.uk).

Digital Object Identifier 10.1109/TWC.2007.05817. of $L$ simultaneous uplink mobile users - each equipped with a single transmit antenna - are received by the $P$ different receiver antennas of the Base Station (BS). At the BS MultiUser Detection (MUD) [5] techniques are invoked for detecting the different users' transmitted signals with the aid of their unique, user-specific spatial signature constituted by their Frequency-Domain CHannel Transfer Functions (FDCHTFs) or, equivalently, Channel Impulse Responses (CIRs). However, in these systems accurate channel estimation is required at the receiver for the sake of invoking both coherent demodulation and interference cancellation [6]. Compared to Single-Input-Single-Output (SISO) systems, channel estimation in the MIMO scenario becomes more challenging, since a significantly increased number of independent transmitterreceiver channel links have to be estimated simultaneously for each subcarrier. Moreover, the interfering signals of the other transmit antennas have to be suppressed.

In the literature, a number of blind channel estimation techniques have been proposed for MIMO-OFDM systems [7][12]. However, most of these approaches suffer from either a slow convergence rate or a performance degradation, owing to the inherent limitations of blind search mechanisms. By contrast, the techniques benefiting from explicit training with the aid of known reference/pilot signals, are typically capable of achieving a better performance at the cost of a reduced effective system throughput. For example, Li et al. [13] proposed an approach of exploiting both transmitter diversity and the delay profile characteristics of typical mobile channels, which was further simplified and enhanced in [14], [15] and [16], respectively. Other schemes employed Minimum Mean Square Error (MMSE) [17], Constrained Least Squares (CLS) [18], iterative Least Squares (LS) [19], SecondOrder Statistics (SOS) based SubSpace (SS) [20] estimation algorithms as well as the QR Decomposition combined with the M-algorithm (QRD-M) [21], [22] or techniques based on Time Of Arrivals (TOAs) [23], etc. Some researchers have their attention focused on designing optimum training patterns or structures [14], [24]. Furthermore, various joint approaches combining channel estimation with data symbol detection at the receiver were also proposed for Code Division Multiple Access (CDMA) [12], [21], SISO OFDM [25]-[27] and MIMO OFDM [22], [28] systems. However, in the context of BLAST or SDMA type multi-user MIMO OFDM systems, all channel estimation techniques found in the literature were developed under the assumption that the number of users $L$ is lower than [7]-[9], [17], [20] or equal to [10], [18], [22]- 
[24], [28] the number of receiver antennas $P$. This assumption is critical for the following reasons. When we have $L>P$, which we refer to as a rank-deficient scenario, the $(P \times L)$ dimensional MIMO channel matrix representing the $P \times L$ channel links becomes singular, thus rendering the degree of freedom of the detector insufficient. This will catastrophically degrade the performance of numerous known detection approaches, such as for example the MMSE algorithm of [4], [5] and the QRD-M algorithm of [22]. Furthermore, the associated significant degradation of the MUD's performance in this rank-deficient scenario will inevitably result in severe error propagation in decision-directed type channel estimators [4].

Against this background, in this paper we propose a new Genetic Algorithm (GA) assisted iterative Joint Channel Estimation and multi-user detection (GA-JCEMUD) approach for multi-user MIMO SDMA-OFDM systems, which provides an effective solution to the multi-user MIMO channel estimation problem in the above-mentioned rank-deficient scenario. Our ambitious goal of supporting a high number of users is possible, because the proposed GA-based technique dispenses with any constraints concerning the rank of the channel matrix. In the literature, only a few channel estimation schemes were proposed based on GAs. More specifically, Yen et al. [29] proposed a GA-aided multi-user CDMA single-antenna receiver, which jointly estimates the transmitted symbols and fading channel coefficients of all the users. A batch blind equalization scheme based on Maximum Likelihood (ML) concatenated channel and data estimation employing a micro genetic algorithm ( $\mu \mathrm{GA})$ and the Viterbi Algorithm (VA) was proposed in [30]. In [31], GA-based approaches were used for finding optimum training sequences for channel estimation in OFDM systems. However, to our best knowledge, no techniques employing GAs for joint channel and data optimization can be found in the literature in the context of multi-user MIMO OFDM. Furthermore, at the time of writing the GAs invoked in the data detection literature [29], [32][38] can only provide a hard-decision output for the Forward Error Correction (FEC) or channel decoder, which inevitably limits the system's achievable performance. By contrast, our proposed GA is capable of providing "soft" outputs and hence it becomes capable of achieving an improved performance with the aid of FEC decoders.

The structure of this paper is as follows. The overview of the proposed scheme is provided in Section II, followed by our detailed analysis in Section III. The numerical results are presented in Section IV, while our final conclusions are offered in Section V.

\section{System OVERVIEW}

In uplink SDMA-OFDM systems, at the $k^{t h}$ subcarrier of the $n^{t h}$ OFDM symbol received by the $P$-element receiver antenna array we have the received complex signal vector $\mathbf{x}[n, k]$, which is constituted by the superposition of the faded signals associated with the $L$ mobile users and contaminated by the Additive White Gaussian Noise (AWGN), expressed as:

$$
\mathbf{x}[n, k]=\mathbf{H}[n, k] \mathbf{s}[n, k]+\mathbf{n}[n, k],
$$

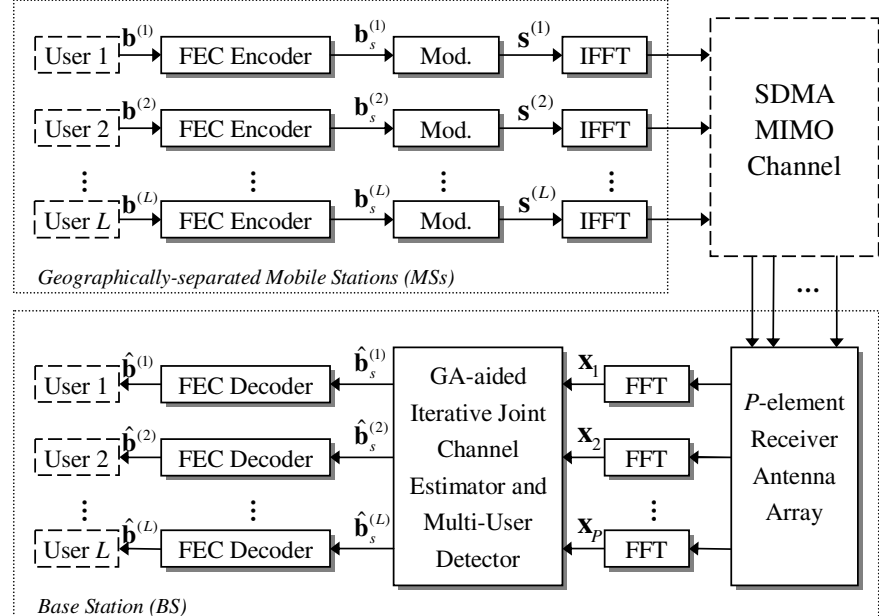

Fig. 1. Schematic of the SDMA-OFDM uplink system employing the proposed GA-aided iterative joint channel estimator and multi-user detector.

where $\mathbf{s}[n, k]$ and $\mathbf{n}[n, k]$ are the transmitted and noise signal vectors, respectively, while $\mathbf{H}[n, k]$ is the FD-CHTF matrix. Note that the mobile users are assumed to be synchronized, whilst the fading envelope of the $(P \times L)$ user-receiver channel links are assumed to be uncorrelated. Figure 1 shows a detailed schematic of a SDMA-OFDM uplink system using the proposed iterative GA-JCEMUD. As shown in the upper half of Figure 1, the information bit blocks $\mathbf{b}^{(l)}(l=1, \cdots, L)$ of the $L$ mobile users are first encoded by the $L$ independent FEC encoders. The resultant coded bits $\mathbf{b}_{s}^{(l)}$ are then mapped to Quadrature Amplitude Modulation (QAM) or Phase-Shift Keying (PSK) symbols $\mathbf{s}^{(l)}$, which are modulated by the Inverse Fast Fourier Transform (IFFT) based OFDM modulators and transmitted over the SDMA MIMO channel. At the BS illustrated at the lower half of Figure 1, the received signal constituted by the noise-contaminated superposition of all users' transmitted signals is OFDM-demodulated at the $P$ receiver antenna elements and forwarded to the iterative GAJCEMUD for joint channel estimation and symbol detection, as it will be detailed in Section III. Then the detected soft bits $\hat{\mathbf{b}}_{s}^{(l)}$ are generated, which are forwarded to the $L$ independent FEC decoders for channel decoding.

\section{GA-ASSisted ITERATIVE JOINT CHANNEL ESTIMATION AND DATA DETECTION}

The proposed iterative GA-JCEMUD is illustrated in Figure 2. We assume that each OFDM symbol consists of $K$ subcarriers. The first transmitted OFDM symbol of all the $L$ users is completely filled by known pilot Quadrature Amplitude Modulation (QAM) symbols. Within the first OFDM symbol duration $(n=0)$, the $\mathrm{BS}$ pilot controller seen in the middle of Figure 2 feeds the pilots to the GA-JCEMUD printed in grey, which simultaneously processes the received signals $x_{p}[0, k](p=1, \cdots, P ; k=1, \cdots, K)$ at the $P$ receiver antenna elements. In order to simplify the analysis, we now focus our attention on the $p^{t h}$ receiver. Based on the pilots and the corresponding received signals, the initial estimates of the FD-CHTFs $\tilde{H}_{p}^{(l)}[0, k](l=1, \cdots, L ; k=1, \cdots, K)$ can 


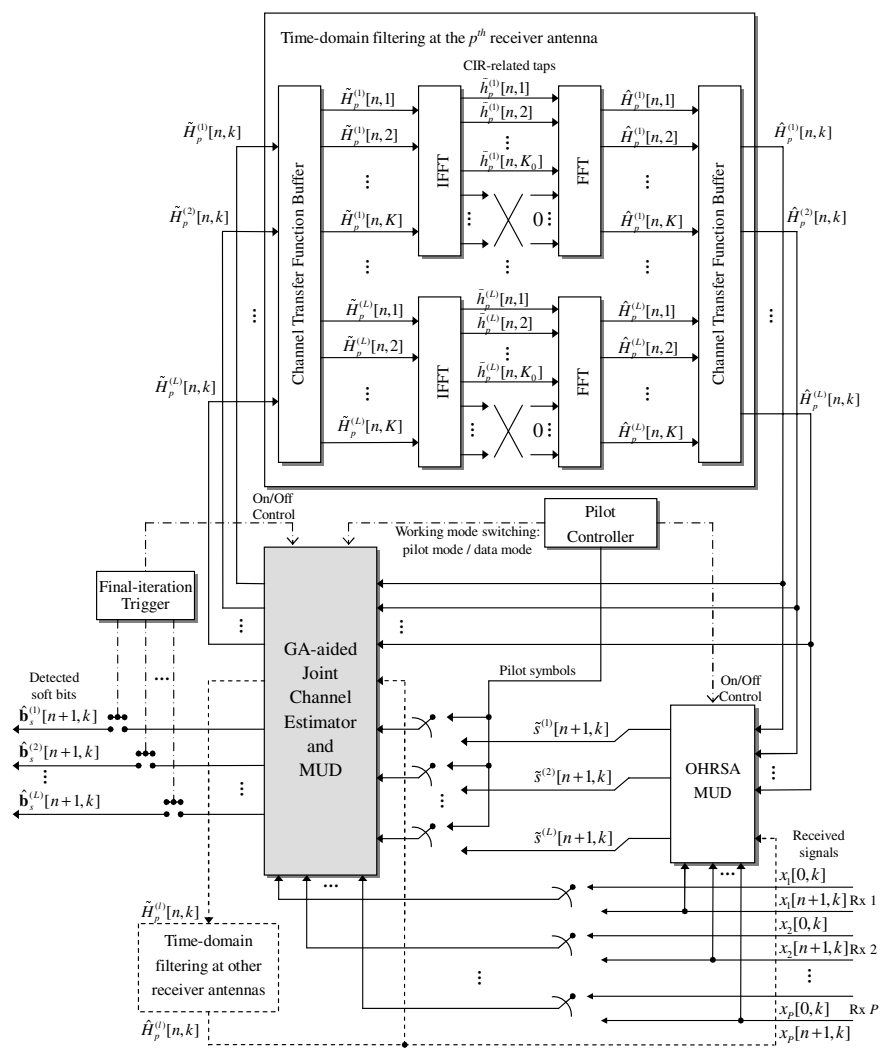

Fig. 2. Structure of the proposed GA-aided iterative joint channel estimator and multi-user detector.

be generated, followed by the time-domain filtering invoking at the $p^{t h}$ receiver, as plotted on the top of Figure 2.

The time-domain filtering is conducted for each of the $L$ users on an OFDM symbol basis. More generally, for the $l^{\text {th }}$ user, the $K$ initial FD-CHTF estimates $\tilde{H}_{p}^{(l)}[n, k](k=$ $1, \cdots, K)$ associated with the current, i.e. the $n^{\text {th }}$ OFDM symbol are processed by a $K$-length Inverse Fast Fourier Transform (IFFT), resulting in the set of $K$ uncorrelated CIR-related taps $\tilde{h}_{p}^{(l)}[n, k]$. Then, only the first $K_{0}$ CIR tap coefficients are retained with the rest set to zero. The value of $K_{0}$ depends on the delay profile of the channel, which is not known a priori at the receiver. However, in many application scenarios it is possible to appropriately over-estimate $K_{0}$ on the basis of previous field experiments. Hence, in our system the value of $K_{0}$ is set to a sufficiently large number so that it significantly exceeds the actual maximum delay spread of the channel. Nonetheless, we point out that the 'significant-tap catching' approaches of [13] can be employed for improving the channel estimator's performance by further removing the low-power taps within the range of $\left[1, \cdots, K_{0}\right]$ according to a pre-defined threshold. The retained CIR-related coefficients $\tilde{h}_{p}^{(l)}[n, k]\left(k=1, \cdots, K_{0}\right)$ are then converted to the improved a posteriori FD-CHTF estimates $\hat{H}_{p}^{(l)}[n, k](k=1, \cdots, K)$ by the Fast Fourier Transform (FFT).

If in the $(n+1)^{t h}$ OFDM symbol duration a data symbol rather than a pilot symbol was transmitted, the pilot controller will enable a first-stage MUD for generating reference symbol estimates. Specifically, the Optimized Hierarchy Reduced Search Algorithm (OHRSA) aided MUD of [39], [40] is employed. With the aid of the a posteriori FD-CHTF estimates $\hat{H}_{p}^{(l)}[n, k]$ associated with the previous OFDM symbol, a subcarrier-by-subcarrier based detection is invoked by the OHRSA MUD for generating an initial guess of the $L$ users' transmitted symbols $s^{(l)}[n+1, k] \quad(l=1, \cdots, L ; k=$ $1, \cdots, K)$. More details of the OHRSA MUD are provided in Section III-B. The resultant symbol estimates $\tilde{s}^{(l)}[n+1, k]$, the FD-CHTF estimates $\hat{H}_{p}^{(l)}[n, k]$ as well as the corresponding received signals $x_{p}[n+1, k](p=1, \cdots, P ; k=1, \cdots, K)$ are then forwarded to the GA-JCEMUD, where the FD-CHTFs and data symbols associated with the $(n+1)^{t h}$ OFDM symbol are jointly optimized on a subcarrier-by-subcarrier basis, as to be discussed in Section III-C. The GA-optimized FD-CHTF estimates $\tilde{H}_{p}^{(l)}[n+1, k]$ are then forwarded to the time-domain filters for further enhancement. Based on the improved channel estimates, the OHRSA MUD is now capable of providing a better initial guess of the transmitted symbols for the GAJCEMUD. This decision-directed process can be invoked for a number of iterations for further performance enhancement. After the final iteration, the $L$ users' detected soft bits $\hat{b}_{s}^{(l)}[n+$ $1, k](l=1, \cdots, L ; k=1, \cdots, K)$ corresponding to the $(n+1)^{t h}$ OFDM symbol are generated.

\section{A. Pilot-aided Initial Channel Estimation}

In order to obtain an initial estimate of the FD-CHTFs, each user's pilot OFDM symbol is multiplied by a user-specific spreading code before it is transmitted. An unspread pilot OFDM symbol constituted by $K$ identical unspread QAM subcarrier symbols can be used by all users, which is known at the receivers. Note that no spreading is applied to the data OFDM symbols. With the aid of the spread pilot OFDM symbols, an initial FD-CHTF estimate is attainable at the receivers, where the Multi-User Interference (MUI) is effectively reduced proportionately to the spreading factor. In our system, orthogonal Walsh-Hadamard-Transform (WHT) [4] based spreading codes of length $L$ are used.

Depending on the specific performance-versus-throughput design tradeoff targeted, this process of generating initial channel estimates can be invoked at pre-defined time intervals. Here we denote the pilot overhead as $\epsilon$, which is defined by the ratio of the number of pilot OFDM symbols to the total number of transmitted OFDM symbols. We will show in Section IV that a good performance is achievable by the proposed scheme with a small value of $\epsilon$.

\section{B. Generating Initial Symbol Estimates}

As mentioned earlier, for each subcarrier, an initial symbol estimate is first obtained with the aid of the first-stage Optimized Hierarchy Reduced Search Algorithm (OHRSA) assisted MUD [39], [40], which exploits the a posteriori FDCHTF estimates generated within the previous OFDM symbol duration. For the sake of notational convenience, in this section the index of $[n, k]$ is omitted. However, we note that the following analysis is conducted on a subcarrier basis.

It is well known that the optimum ML MUD [4] employs an exhaustive search for finding the most likely transmitted signals. More explicitly, the $L$-user symbol vector estimate 
$\hat{\mathrm{S}}_{\mathrm{ML}}$ can be obtained by minimizing the following metric:

$$
\hat{\mathbf{s}}_{\mathrm{ML}}=\arg \left\{\min _{\tilde{\mathbf{s}} \in \mathcal{M}^{L}}\|\mathbf{x}-\mathbf{H} \check{\mathbf{s}}\|^{2}\right\}
$$

where $\check{\mathbf{s}}$ is an a priori candidate vector of the set $\mathcal{M}^{L}$, which is constituted by $2^{m L}$ trial-vectors, where $m$ denotes the number of Bits Per Symbol (BPS). More specifically, $\mathcal{M}^{L}$ is formulated as:

$$
\begin{aligned}
& \mathcal{M}^{L}= \\
& \left\{\check{\mathbf{s}}=\left(\check{s}^{(1)}, \check{s}^{(2)}, \cdots, \check{s}^{(L)}\right)^{T} \mid \check{s}^{(1)}, \check{s}^{(2)}, \cdots, \check{s}^{(L)} \in \mathcal{M}_{c}\right\},
\end{aligned}
$$

where $\mathcal{M}_{c}$ denotes the set containing the $2^{m}$ legitimate complex constellation points associated with the specific modulation scheme employed. Furthermore, it can be shown that Equation (2) is equivalent to [39], [40]:

$$
\hat{\mathbf{s}}=\arg \left\{\min _{\check{\mathbf{s}} \in \mathcal{M}^{L}}\left\|\mathbf{V}\left(\check{\mathbf{s}}-\hat{\mathbf{s}}_{\mathrm{MMSE}}\right)\right\|^{2}\right\},
$$

where $\mathrm{V}$ is an upper-triangular matrix having positive realvalued elements on the main diagonal and satisfying

$$
\mathbf{V}^{H} \mathbf{V}=\mathbf{H}^{H} \mathbf{H}+\sigma_{n}^{2} \mathbf{I},
$$

while

$$
\hat{\mathbf{s}}_{\mathrm{MMSE}}=\left(\mathbf{H}^{H} \mathbf{H}+\sigma_{n}^{2} \mathbf{I}\right)^{-1} \mathbf{H}^{H} \mathbf{x}
$$

is the unconstrained MMSE-based estimate of the transmitted signal vector $\mathbf{s}$, with $\mathbf{I}$ and $\sigma_{n}^{2}$ are the identity matrix and the AWGN noise variance, respectively. The superscript of $(\cdot)^{H}$ denotes the Hermitian transpose. Since $\mathbf{V}$ is an uppertriangular matrix, a specific cost function can be derived:

$$
\begin{aligned}
\Phi(\check{\mathbf{s}}) & =\left\|\mathbf{V}\left(\check{\mathbf{s}}-\hat{\mathbf{s}}_{\mathrm{MMSE}}\right)\right\|^{2} \\
& =\left(\check{\mathbf{s}}-\hat{\mathbf{s}}_{\mathrm{MMSE}}\right)^{H} \mathbf{V}^{H} \mathbf{V}\left(\check{\mathbf{s}}-\hat{\mathbf{s}}_{\mathrm{MMSE}}\right) \\
& =\sum_{i=1}^{L} \mid \sum_{j=i}^{L} v_{i j}\left(\check{s}^{(j)}-\left.\hat{s}_{\mathrm{MMSE}}^{(j)}\right|^{2}\right. \\
& =\sum_{i=1}^{L} \phi_{i}\left(\check{\mathbf{s}}_{i}\right),
\end{aligned}
$$

where $\phi_{i}\left(\check{\mathbf{s}}_{i}\right)$ is a set of sub-cost functions, and $\check{\mathbf{s}}_{i}$ represents the sub-vectors of $\check{\mathbf{s}}$, formulated as:

$$
\check{\mathbf{s}}_{i}=\left[\check{s}^{(i)}, \check{s}^{(i+1)}, \cdots, \check{s}^{(L)}\right], \quad i \in\{1, \cdots, L\} .
$$

Note that the outputs of both $\Phi(\check{\mathbf{s}})$ and $\phi_{i}\left(\check{\mathbf{s}}_{i}\right)$ are real-valued. Furthermore, we have:

$$
\begin{aligned}
\phi_{i}\left(\check{\mathbf{s}}_{i}\right) & =\left|\sum_{j=i}^{L} v_{i j}\left(\check{s}^{(j)}-\hat{s}_{\mathrm{MMSE}}^{(j)}\right)\right|^{2} \\
& =\left|v_{i i}\left(\check{s}^{(i)}-\hat{s}_{\mathrm{MMSE}}^{(i)}\right)+\sum_{j=i+1}^{L} v_{i j}\left(\check{s}^{(j)}-\hat{s}_{\mathrm{MMSE}}^{(j)}\right)\right|^{2}(9)
\end{aligned}
$$

Based on Equation (9), $\Phi(\check{\mathbf{s}})$ can be re-defined as the Cumulative Sub-Cost (CSC) function:

$$
\begin{aligned}
\Phi_{L}\left(\check{\mathbf{s}}_{L}\right) & =\phi_{L}\left(\check{\mathbf{s}}_{L}\right)=\left|v_{L L}\left(\check{s}^{(L)}-\hat{s}_{\mathrm{MMSE}}^{(L)}\right)\right|^{2}, i=L \\
\Phi_{i}\left(\check{\mathbf{s}}_{i}\right) & =\Phi_{i+1}\left(\check{\mathbf{s}}_{i+1}\right)+\phi_{i}\left(\check{\mathbf{s}}_{i}\right), i=L-1, \cdots, 1 .
\end{aligned}
$$

The Euclidean norm of Equation (10) can be interpreted as a weighted Euclidean distance between the candidate constellation point $\check{s}^{(l)}$ and the unconstrained MMSE estimate $\hat{s}_{\text {MMSE }}^{(l)}$ of the transmitted signal component $s_{\mathrm{MMSE}}^{(l)}$. Explicitly, the CSC functions obey the property:

$$
\Phi(\check{\mathbf{s}})=\Phi_{1}\left(\check{\mathbf{s}}_{1}\right)>\Phi_{2}\left(\check{\mathbf{s}}_{2}\right)>\cdots>\Phi_{L}\left(\check{\mathbf{s}}_{L}\right)>0
$$

for all possible combinations of $\check{\mathbf{s}} \in \mathcal{M}^{L}$ and $\hat{\mathbf{s}}_{\text {MMSE }} \in \mathbb{C}^{L}$, where the $L$-dimensional complex space $\mathbb{C}^{L}$ contains all possible unconstrained MMSE estimates $\hat{\mathrm{S}}_{\mathrm{MMSE}}$ of the transmitted signal vector $\mathbf{s}$.

Exploiting the monotonously increasing nature of the nonbinary, i.e. multi-bit symbol based CSC functions of Equation (11), a bit-based recursive search algorithm can be developed [39], [40], where the $\left(L_{b}=m L\right)$-dimensional bit vectors b constituting the $L$ users' bits, rather than the symbol vectors $\check{\mathrm{s}}$ are used as the candidates for the CSC functions given by Equations (7) and (10). More specifically, two legitimate hypotheses of -1 and 1 are stipulated at each recursive step $i$ of the search algorithm, concerning one of the bits of the bit-based trial vector $\check{\mathbf{b}}_{i}$. This allows us now to interpret the CSC functions as the Euclidian distance contribution of the specific $\check{\mathbf{b}}_{i}$, when considering a specific bit of a given symbol of a given user. The recursive search process commences with the evaluation of the CSC function of Equation (10a), followed by the calculation of the conditioned CSC function values of Equation (10b). Moreover, for each tentatively assumed value of $\check{\mathbf{b}}_{i}$ a successive recursive search step $(i-1)$ is invoked, which is conditioned on the hypotheses made in all preceding recursive steps $j=i, \cdots, L_{b}=m L$. Upon each arrival at the index $i=1$ of the recursive process, a complete bit-based candidate vector $\check{\mathbf{b}}$ associated with a certain symbol vector $\check{\mathbf{s}}$ is hypothesized and the corresponding value of the cost function $\Phi(\check{\mathbf{b}})$ formulated in Equation (7) is evaluated. Furthermore, with the aid of a carefully-designed search strategy [39], [40], the OHRSA is capable of arriving at the optimum ML estimate at a significantly reduced complexity. For more details on the OHRSA MUD, the interested reader is referred to [39], [40].

\section{GA-aided Joint FD-CHTF and Data Optimization Provid- ing Soft Outputs}

1) Joint Genetic Optimization: In comparison to the pure GA-based MUDs [33]-[35], [37], [38], which optimize the multi-user data symbols only, the joint optimization work requires the FD-CHTFs to be simultaneously optimized along with the data symbols, as in [29], [32].

Furthermore, concerning the MIMO channel's structure, the GA individuals' representation of [29], [32] is extended to:

$$
\left\{\begin{array}{c}
\tilde{\mathbf{s}}_{(y, x)}[n, k]= \\
\quad\left[\begin{array}{cccc}
\tilde{s}_{(y, x)}^{(1)}[n, k], & \tilde{s}_{(y, x)}^{(2)}[n, k], \cdots, & \left.\tilde{s}_{(y, x)}^{(L)}[n, k]\right]
\end{array}\right. \\
\tilde{\mathbf{H}}_{(y, x)}[n, k]= \\
{\left[\begin{array}{cccc}
\tilde{H}_{1,(y, x)}^{(1)}[n, k] & \tilde{H}_{1,(y, x)}^{(2)}[n, k] & \cdots & \tilde{H}_{1,(y, x)}^{(L)}[n, k] \\
\tilde{H}_{2,(y, x)}^{(1)}[n, k] & \tilde{H}_{2,(y, x)}^{(2)}[n, k] & \cdots & \tilde{H}_{2,(y, x)}^{(L)}[n, k] \\
\vdots & \vdots & \ddots & \vdots \\
\tilde{H}_{P,(y, x)}^{(1)}[n, k] & \tilde{H}_{P,(y, x)}^{(2)}[n, k] & \cdots & \tilde{H}_{P,(y, x)}^{(L)}[n, k]
\end{array}\right]}
\end{array}\right.
$$


in the context of the $k^{\text {th }}$ subcarrier of the $n^{\text {th }}$ OFDM symbol, where the subscript $(y, x)$ denotes the $x^{\text {th }}(x=1, \cdots, X)$ individual at the $y^{\text {th }}(y=1, \cdots, Y)$ generation. Note that we have $\tilde{s}_{(y, x)}^{(l)}[n, k] \in \mathcal{M}_{c}$ and $\tilde{H}_{p,(y, x)}^{(l)}[n, k] \in \mathbb{C}$, respectively, where $\mathbb{C}$ denotes the set of all complex numbers. During the stage of initialization, the GA generates a population of $X$ individuals represented by Equation (12), based on the initial FD-CHTF estimates of Section III-A and the initial symbol estimates of Section III-B. More explicitly, at the $k^{\text {th }}$ subcarrier in the $(n+1)^{\text {th }}$ OFDM symbol duration, the $(y, x)=(1,1)^{s t}$ individual is generated as:

$$
\left\{\begin{array}{c}
\tilde{s}_{(1,1)}^{(l)}[n+1, k]=\tilde{s}^{(l)}[n+1, k] \\
\tilde{H}_{p,(1,1)}^{(l)}[n+1, k]=\tilde{H}_{p}^{(l)}[n, k] \\
l=1, \cdots, L ; p=1, \cdots, P,
\end{array}\right.
$$

where $\tilde{s}^{(l)}[n+1, k]$ represents the initial symbol estimates provided by the OHRSA MUD, while $\tilde{H}_{p}^{(l)}[n, k]$ denotes the initial FD-CHTF estimates associated with the previous, i.e. the $n^{\text {th }}$ OFDM symbol. The other $X-1$ individuals are then created by the GA's mutation operator [32]:

$$
\left\{\begin{array}{c}
\tilde{s}_{(y, x)}^{(l)}[n+1, k]=\operatorname{MUTATE}\left[\begin{array}{l}
\left.\tilde{s}_{(1,1)}^{(l)}[n+1, k]\right] \\
\tilde{H}_{p,(y, x)}^{(l)}[n+1, k]=\operatorname{MUTATE}\left[\tilde{H}_{p,(1,1)}^{(l)}[n+1, k]\right] \\
x=2 \ldots X ; y=1 \ldots Y ; l=1 \ldots L ; p=1 \ldots P .
\end{array},\right.
\end{array}\right.
$$

After the creation of the $y=1^{\text {st }}$ generation, which consists of the population of the initially generated $X$ individuals, the GA-based search process can be invoked for jointly optimizing the estimates of the multi-user symbols and FD-CHTFs.

The basic idea of the GA-based optimization is to find the optimum or a near-optimum solution according to a predefined objective function $(O F)$. In the context of the joint detection problem in SDMA-OFDM systems, the GA's OF can be based on the ML metric of Equation (2), formulated as:

$$
\Omega(\tilde{\mathbf{H}}[n, k], \tilde{\mathbf{s}}[n, k])=\|\mathbf{x}[n, k]-\tilde{\mathbf{H}}[n, k] \cdot \tilde{\mathbf{s}}[n, k]\|^{2},
$$

where each combination of the trial data vector $\tilde{\mathbf{s}}[n, k]$ and trial FD-CHTF matrix $\tilde{\mathbf{H}}[n, k]$ constitutes a GA individual defined in Equation (12). The output of the $\mathrm{OF}$ is referred to as the objective score (OS), and the individual having a lower OS is considered to have a higher fitness value. Explicitly, the GA's ultimate aim is to find the individual that has the highest fitness value. This is achieved with the aid of the genetic operators invoked during the evolution process, such as cross-over and mutation [32], where specific elements of the different individuals are exchanged and mutated to produce the corresponding offspring, whose fitness values are statistically improved over the consecutive generations. For space economy, the interested reader is referred to [32], [38], where more details of the GA-based MUDs can be found.

Note that since the FD-CHTFs and data symbols are jointly optimized within the same genetic process, the individuals having better FD-CHTF estimates will have a higher probability of producing better symbol estimates and vice versa. From a statistical point of view, this joint optimization is a "self-adaptive" process leading towards the optimum solution, provided that the number of GA generations is sufficiently high. Furthermore, compared to other techniques, for example that of [30], where channel estimation and symbol detection are carried out by the GA and the VA separately, the proposed joint scheme is capable of reducing the associated complexity. More specifically, the GA of [30] can only provide channel estimates, whilst the data symbols are detected by the subsequent VA-based detector. By contrast, thanks to the joint optimization structure, our scheme simultaneously generates both channel and symbol estimates within the same genetic process, implying that no additional complexity is required for data detection.

Moreover, it is worth pointing out that at the time of writing the GA-aided detection schemes found in the literature [29], [32]-[35], [37], [38] are only capable of providing singleindividual based hard-decoded symbol estimates, which inevitably limits the GA-aided system's attainable performance. In Section III-C.2, we will introduce a method, which enables the GA to provide soft outputs based on the entire population.

2) Generating the GA's Soft Outputs: For the sake of notational convenience, the index of $[n, k]$ is omitted in this section. The soft-bit value or Log-Likelihood Ratio (LLR) associated with the $\left(m_{B}\right)^{t h}$ bit position of the $l^{t h}(l=$ $1, \cdots, L)$ user's transmitted symbol $s^{(l)}$ can be formulated as:

$$
\mathcal{L}_{l, m_{B}}=\ln \frac{P\left(b_{l, m_{B}}=1 \mid \mathbf{x}, \mathbf{H}\right)}{P\left(b_{l, m_{B}}=0 \mid \mathbf{x}, \mathbf{H}\right)} .
$$

Note that the probability $P\left(b_{l, m_{B}}=b \mid \mathbf{x}, \mathbf{H}\right)$ that the symbol transmitted by the $l^{t h}$ user has the $\left(m_{B}\right)^{t h}$ bit value of $b_{l, m_{B}}=$ $b \in\{0,1\}$, is given by the sum of all the probabilities of the symbol combinations which assume that $b_{l, m_{B}}=b$. Hence, Equation (16) can be equivalently rewritten as:

$$
\mathcal{L}_{l, m_{B}}=\ln \frac{\sum_{\check{\mathbf{s}} \in \mathcal{M}_{l, m_{B}, 1}^{L}} P(\check{\mathbf{s}} \mid \mathbf{x}, \mathbf{H})}{\sum_{\check{\mathbf{s}} \in \mathcal{M}_{l, m_{B}, 0}^{L}} P(\check{\mathbf{s}} \mid \mathbf{x}, \mathbf{H})},
$$

where $\mathcal{M}_{l, m_{B}, b}^{L}$ denotes the specific subset associated with the $l^{\text {th }}$ user, which is constituted by those specific trial vectors, whose $l^{\text {th }}$ element's $\left(m_{B}\right)^{t h}$ bit has a value of $b$, which is expressed as:

$$
\begin{aligned}
& \mathcal{M}_{l, m_{B}, b}^{L}=\left\{\check{\mathbf{s}}=\left[\check{s}^{(1)}, \check{s}^{(2)}, \cdots, \check{s}^{(L)}\right]^{T} \mid\right. \\
& \left.\left\{\check{s}^{(1)}, \check{s}^{(2)}, \cdots, \check{s}^{(L)} \in \mathcal{M}_{c}\right\} \wedge\left\{b_{l, m_{B}}=b\right\}\right\} .
\end{aligned}
$$

With the aid of Bayes' theorem, we have:

$$
P(\check{\mathbf{s}} \mid \mathbf{x}, \mathbf{H})=P(\mathbf{x} \mid \check{\mathbf{s}}, \mathbf{H}) \frac{P(\check{\mathbf{s}})}{P(\mathbf{x})} .
$$

Upon substituting Equation (19) into Equation (17), we arrive at:

$$
\mathcal{L}_{l, m_{B}}=\ln \frac{\sum_{\check{\mathbf{s}} \in \mathcal{M}_{l, m_{B}, 1}^{L}} P(\mathbf{x} \mid \check{\mathbf{s}}, \mathbf{H})}{\sum_{\check{\mathbf{s}} \in \mathcal{M}_{l, m_{B}, 0}^{L}} P(\mathbf{x} \mid \check{\mathbf{s}}, \mathbf{H})} .
$$

Note that here we have assumed that the different $\left(2^{m}\right)$ -

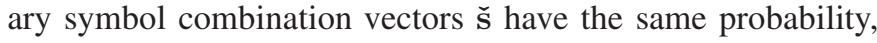
namely that $P(\check{\mathbf{s}}), \check{\mathbf{s}} \in \mathcal{M}_{c}$ is a constant. On the other hand, it can be observed from Equation (1) that $\mathrm{x}$ is a random sample of the $L$-dimensional multi-variate complex Gaussian 
distribution, where the mean vector is $\mathbf{H s}$, while the $(P \times P)$ dimensional covariance matrix $\mathbf{R}_{\mathbf{n}}$ is given by:

$$
\mathbf{R}_{\mathbf{n}}=E\left\{\mathbf{n n}^{H}\right\}=\sigma_{n}^{2} \mathbf{I},
$$

and the noise encountered at the $P$ receiver antennas is assumed to be uncorrelated. Hence, the above-mentioned multivariate complex Gaussian distribution can be described by [4]:

$$
f(\mathbf{x} \mid \mathbf{s}, \mathbf{H})=\frac{1}{\pi^{P}\left|\mathbf{R}_{\mathbf{n}}\right|} \exp \left\{-(\mathbf{x}-\mathbf{H s})^{H} \mathbf{R}_{\mathbf{n}}^{-\mathbf{1}}(\mathbf{x}-\mathbf{H s})\right\} .
$$

When substituting Equation (21) into Equation (22), we have:

$$
f(\mathbf{x} \mid \mathbf{s}, \mathbf{H})=\frac{1}{\pi^{P} \sigma_{n}^{2}} \exp \left\{-\frac{1}{\sigma_{n}^{2}}\|\mathbf{x}-\mathbf{H} \mathbf{s}\|^{2}\right\} .
$$

Note that $f(\mathbf{x} \mid \mathbf{s}, \mathbf{H})=P(\mathbf{x} \mid \mathbf{s}, \mathbf{H})$ is the a priori probability that the vector $\mathbf{x}$ has been received under the condition that the vector $\mathbf{s}$ was transmitted over the MIMO channel characterized by the FD-CHTF matrix $\mathbf{H}$. Thus, Equation (20) can be further developed with the aid of Equation (23), yielding:

$$
\mathcal{L}_{l, m_{B}}=\ln \frac{\sum_{\check{\mathbf{s}} \in \mathcal{M}_{l, m_{B}, 1}^{L}} \frac{1}{\pi^{P} \sigma_{n}^{2}} \exp \left\{-\frac{1}{\sigma_{n}^{2}}\|\mathbf{x}-\mathbf{H} \check{\mathbf{s}}\|^{2}\right\}}{\sum_{\check{\mathbf{s}} \in \mathcal{M}_{l, m_{B}, 0}^{L}} \frac{1}{\pi^{P} \sigma_{n}^{2}} \exp \left\{-\frac{1}{\sigma_{n}^{2}}\|\mathbf{x}-\mathbf{H} \check{\mathbf{s}}\|^{2}\right\}} .
$$

In order to avoid the exponential computation imposed by Equation (24), the maximum-approximation [4] can be applied, yielding:

$$
\mathcal{L}_{l, m_{B}} \approx-\frac{1}{\sigma_{n}^{2}}\left[\left\|\mathbf{x}-\mathbf{H} \check{\mathbf{s}}_{l, m_{B}, 1}\right\|^{2}-\left\|\mathbf{x}-\mathbf{H} \check{\mathbf{s}}_{l, m_{B}, 0}\right\|^{2}\right],
$$

where

$$
\check{\mathbf{s}}_{l, m_{B}, b}=\arg \left\{\min _{\check{\mathbf{s}} \in \mathcal{M}_{l, m_{B}, b}^{L}}\left[\|\mathbf{x}-\mathbf{H} \check{\mathbf{s}}\|^{2}\right]\right\}, \quad b=0,1 .
$$

Furthermore, concerning the fact that the true FD-CHTF matrix $\mathbf{H}$ is unknown and using Equation (15), Equation (25) can be represented as:

$$
\mathcal{L}_{l, m_{B}} \approx-\frac{1}{\sigma_{n}^{2}}\left[\bar{\Omega}_{l, m_{B}, 1}-\bar{\Omega}_{l, m_{B}, 0}\right],
$$

where

$$
\bar{\Omega}_{l, m_{B}, b}=\min \left[\Omega\left(\tilde{\mathbf{H}}, \check{\mathbf{s}}_{l, m_{B}, b}\right), \omega\right], \quad b=0,1,
$$

and $\omega=P \cdot L$ is a normalization factor. Equation (27) suggests that the LLRs can be obtained by evaluating the GA's OF. More explicitly, in order to calculate the LLR of the $\left(m_{B}\right)^{t h}$ bit of the $l^{t h}(l=1, \cdots, L)$ user at the specific subcarrier considered, the $X$ individuals in the GA's final generation are divided into two groups, where the first (or second) group is constituted by those individuals that have a value of one (or zero) at the $\left(m_{B}\right)^{t h}$ bit of the $l^{\text {th }}$ user's estimated transmitted symbol. The resultant lowest OS calculated in each of the two groups is then compared to $\omega$, and the smaller of the two will be used in Equation (27) for calculating the corresponding LLR, which can therefore assist the channel decoder in improving the SDMA-OFDM system's performance.

It is worth pointing out that the proposed GA generating the above-mentioned population-based soft outputs only imposes a modest complexity increase in comparison to the conventional hard-decision aided individual-based GAs [29], [32][35], [37], [38]. This is because the only additional operation required by the proposed scheme besides the calculation of Equation (27), is to compare $\omega$ to the OSs, which are already available, since the results of the OF evaluation carried out by the conventional GAs can be readily used.

\section{Simulation Results}

In this section, we will quantify the performance of the MIMO SDMA-OFDM system using the proposed GA-aided iterative joint channel estimation and multi-user detection technique. Due to the space limitations, we only show the results achieved by the proposed scheme in the rank-deficient scenario, although we point out that it performs equally well in the scenarios, where the number of users $L$ is less than or equal to the number of receiver antenna elements $P$. More specifically, a rank-deficient scenario where $L=4$ users were supported by $P=2$ receiver antenna elements was considered. As an example, a simple two-path Rayleigh fading channel model was employed, where the associated delay profile was $\left(0.7740 \cdot z^{0}+0.6332 \cdot z^{-1}\right)$. The value of the parameter $K_{0}$ was set to $8 \gg 2$, which is potentially capable of tolerating an increase of the actual dispersion up to eight CIR taps. Each of the paths experienced independent Rayleigh fading having the same Doppler frequency of $F_{D}=f_{d} T_{s}$ normalized to the OFDM symbol rate, where $f_{d}$ and $T_{s}$ are the maximum Doppler frequency and the OFDM symbol duration including the cyclic prefix, respectively. The channel was assumed to be OFDM symbol-invariant, implying that the CIR taps were assumed to be constant for the duration of one OFDM symbol, but they were faded at the beginning of each symbol. Each user's associated transmit power or signal variance was assumed to be unity. Moreover, both scenarios with and without FEC coding were investigated. In the FECcoded scenario, as an example, a half-rate binary Low Density Parity Check (LDPC) [41] code was employed. However, other FEC codes, for example Turbo Convolutional (TC) codes are also applicable to the proposed system. For the reader's convenience, the simulation parameters are summarized in Table I. For more details on the GA's configuration, the interested reader is referred to Section III-C.1 and [32], [37], [38].

In Figure 3 we compare the Bit Error Ratio (BER) versus Signal-to-Noise Ratio (SNR) performance of both the uncoded and LDPC-coded GA-JCEMUD/SDMA-OFDM systems in conjunction with different values of the OFDM symbol normalized Doppler frequency $F_{D}$. The performances of the systems employing the linear MMSE MUD or the optimum ML MUD are also provided as references, both assuming perfect Channel State Information (CSI). A pilot overhead of $\epsilon=2.5 \%$ was assumed and the GA-JCEMUD used a single iteration. As shown in Figure 3, unsurprisingly, the performances of both the uncoded and coded GA-JCEMUD aided systems degraded, when $F_{D}$ was increased, since a higher Doppler frequency implies that the channel fades more rapidly, which renders channel estimation more challenging. This is especially true for MIMO systems, even more so for rank-deficient MIMO systems, as discussed in Section I. 
TABLE I

BASIC Simulation PARAMETERS USED IN SECTION IV.

\begin{tabular}{|c|c|c|c|}
\hline \multirow{5}{*}{$\begin{array}{c}\text { LDPC } \\
\text { parameters }\end{array}$} & \multicolumn{2}{|l|}{ Modem } & 4QAM \\
\hline & \multicolumn{2}{|l|}{ Code rate } & $1 / 2$ \\
\hline & \multicolumn{2}{|l|}{ Column weight } & 2.5 \\
\hline & \multicolumn{2}{|l|}{ Maximum iterations } & 10 \\
\hline & \multicolumn{2}{|c|}{ Block length of input bits } & 640 bits \\
\hline \multirow{16}{*}{$\begin{array}{c}\text { GA-JCEMUD } \\
\text { parameters }\end{array}$} & \multicolumn{2}{|l|}{ Symbol initialization } & OHRSA [39], [42] \\
\hline & \multicolumn{2}{|c|}{ Mating pool creation strategy } & Pareto-Optimality [43] \\
\hline & \multicolumn{2}{|l|}{ Selection method } & Fitness-Proportionate [32] \\
\hline & \multirow[t]{2}{*}{ Cross-over scheme } & FD-CHTF & Blend cross-over [44] \\
\hline & & Symbol & Uniform cross-over [32] \\
\hline & \multirow[t]{2}{*}{ Mutation scheme } & FD-CHTF & Step mutation [29], [32] \\
\hline & & Symbol & Uniform mutation [32] \\
\hline & \multirow[t]{2}{*}{ Mutation probability } & FD-CHTF & 0.20 \\
\hline & & Symbol & 0.15 \\
\hline & \multicolumn{2}{|c|}{ Elitism [32] percentage } & $10 \%$ \\
\hline & \multicolumn{2}{|l|}{ Incest prevention [32] } & Enabled \\
\hline & \multicolumn{2}{|l|}{ Population size } & 40 \\
\hline & \multicolumn{2}{|l|}{ Generations } & 5 \\
\hline & \multicolumn{2}{|l|}{ GA's output } & Population-based \\
\hline & \multicolumn{2}{|l|}{ Iterations } & 1 (unless specified) \\
\hline & \multicolumn{2}{|l|}{ Pilot overhead $\epsilon$} & $2.5 \%$ (unless specified) \\
\hline \multirow{6}{*}{$\begin{array}{l}\text { Channel } \\
\text { parameters }\end{array}$} & \multicolumn{2}{|l|}{ Paths } & 2 \\
\hline & \multicolumn{2}{|l|}{ Delay profile } & $0.7740 \cdot z^{0}+0.6332 \cdot z^{-1}$ \\
\hline & \multicolumn{2}{|l|}{$K_{0}$} & 8 \\
\hline & \multicolumn{2}{|l|}{ Subcarriers $K$} & 64 \\
\hline & \multicolumn{2}{|l|}{ Cyclic prefix } & 8 \\
\hline & \multicolumn{2}{|l|}{$F_{D}$} & 0.003 (unless specified) \\
\hline
\end{tabular}

Nonetheless, with only a $2.5 \%$ pilot overhead, the proposed GA-JCEMUD/SDMA-OFDM system was capable of achieving a performance close to the perfect-CSI aided optimum ML MUD at $F_{D}=0.001$. By contrast, the system employing the MMSE MUD completely failed even with the aid of perfect CSI, owing to the insufficient degree of detection freedom experienced in rank-deficient scenarios. On the other hand, a significant iteration gain was achievable, when the GAJCEMUD invoked more iterations, as revealed by Figure 4, where we had $\epsilon=2.5 \%$ and $F_{D}=0.003$. Furthermore, the performance of the uncoded system consistently improved as the number of iterations was increased, while in the LDPCcoded system most of the gain was attained by the first GAJCEMUD iteration.

In Figure 5, we provide the average channel Mean Square Error (MSE) performance of the GA-JCEMUD/SDMAOFDM system. The performance of the reference system employing $\epsilon=100 \%$ pilot overhead is also provided. The average MSE is defined by:

$$
\overline{\mathrm{MSE}}=\frac{1}{N_{T}} \sum_{n=1}^{N_{T}} \overline{\operatorname{MSE}}[n]
$$

where $N_{T}$ is the total number of OFDM symbols transmitted, while $\overline{\mathrm{MSE}}[n]$ is the average MSE associated with the FD-
CHTFs of the $n^{t h}$ OFDM symbol, given by:

$$
\overline{\mathrm{MSE}}[n]=\frac{1}{P L K} \sum_{p=1}^{P} \sum_{l=1}^{L} \sum_{k=1}^{K}\left|\hat{H}_{p}^{(l)}[n, k]-H_{p}^{(l)}[n, k]\right|^{2} .
$$

Here we point out that the MSE performance of the GAJCEMUD is the same both with and without employing FEC coding. This is because the GA-aided joint optimization process has no direct interaction with the outer FEC code and thus it becomes independent of the codec. Observe from Figure 5 that as expected, the MSE performance was improved when the number of GA-JCEMUD iterations was increased. Moreover, when the SNR exceeded about $13 \mathrm{~dB}$, the GAJCEMUD using $\epsilon=2.5 \%$ pilot overhead approached the bestcase performance associated with $\epsilon=100 \%$.

As a further investigation, a visual comparison of the true and estimated FD-CHTFs is portrayed in Figure 6. More specifically, the $L$ users' FD-CHTFs associated with a specific receiver antenna element during a block of 40 consecutive OFDM symbols are plotted at a SNR value of $20 \mathrm{~dB}$. Each dot of the curves plotted in Figure 6 represents a complex-valued FD-CHTF at a specific subcarrier. By observing the perfect channel-knowledge based illustration at the top of Figure 6, we can see that the FD-CHTF at each subcarrier evolves over the duration of the 40 OFDM symbols, where the thickness of the ring-shaped formations indicates the amount of FD$\mathrm{CHTF}$ change during this time interval. The full perimeter of 
LDPC-GA-JCEMUD-SDMA-OFDM, L4/P2, 4QAM, 2-path Rayleigh

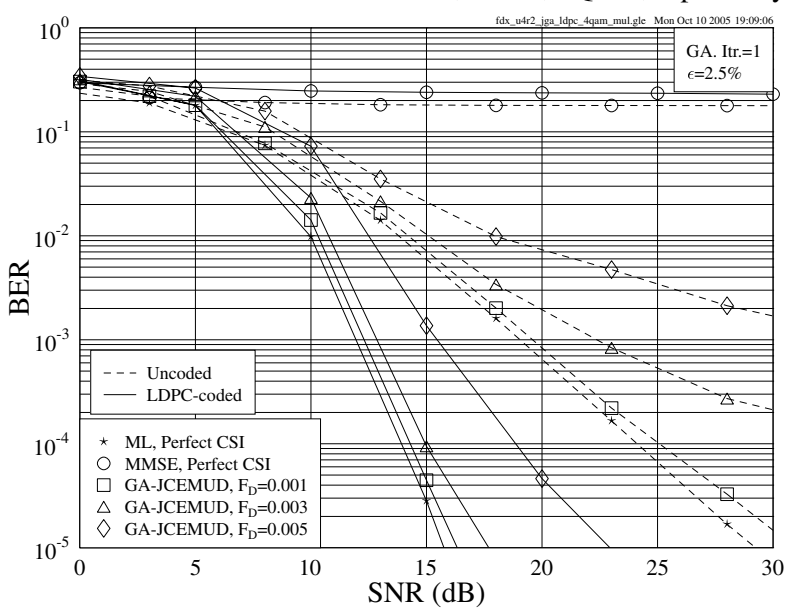

Fig. 3. BER versus SNR performances of the uncoded and LDPCcoded iterative GA-JCEMUD assisted SDMA-OFDM systems in the rank-deficient scenario, where $L=4$ users were supported with the aid of $P=2$ receiver antenna elements, while assuming different values of the OFDM symbol normalized Doppler frequency $F_{D}$. The basic simulation parameters are given in Table I.

LDPC-GA-JCEMUD-SDMA-OFDM, L4/P2, 4QAM, 2-path Rayleigh

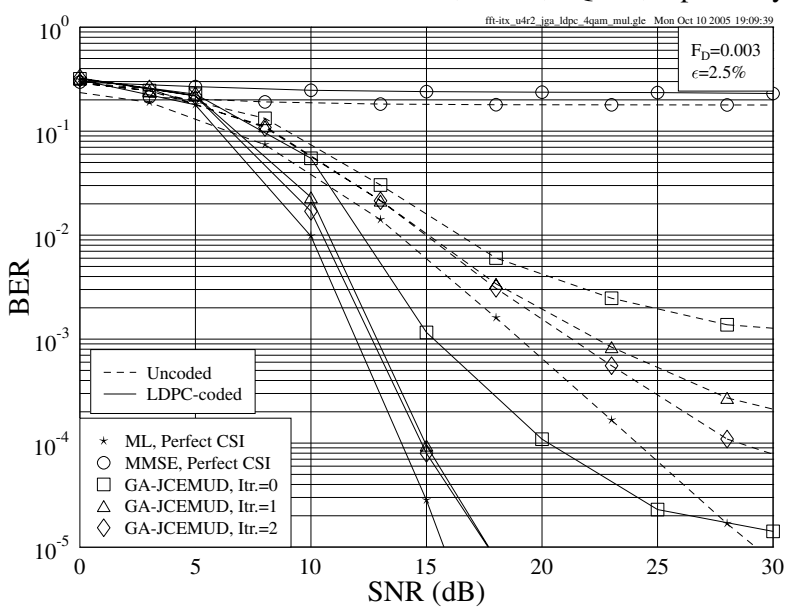

Fig. 4. BER versus SNR performances of the uncoded and LDPCcoded iterative GA-JCEMUD assisted SDMA-OFDM systems in the rank-deficient scenario, where $L=4$ users were supported with the aid of $P=2$ receiver antenna elements, while invoking different numbers of GA-JCEMUD iterations. The basic simulation parameters are given in Table I.

the ring is constituted by the $K=64$ spoke-like formations corresponding to the 64 OFDM subcarriers. Explicitly, the radiuses of the FD-CHTF rings associated with the four user-receiver channel links are significantly different. This is because each individual link is subjected to independent fading, and although the Doppler frequency encountered at the four links is identical, their short-term envelope fluctuation observed over the 40 OFDM symbol durations is different. However, by comparing the subfigures at the top and bottom of Figure 6, we can see that the FD-CHTF estimates closely match their true values, resulting in a similar FD-CHTF contour for each of the four channel links. This implies

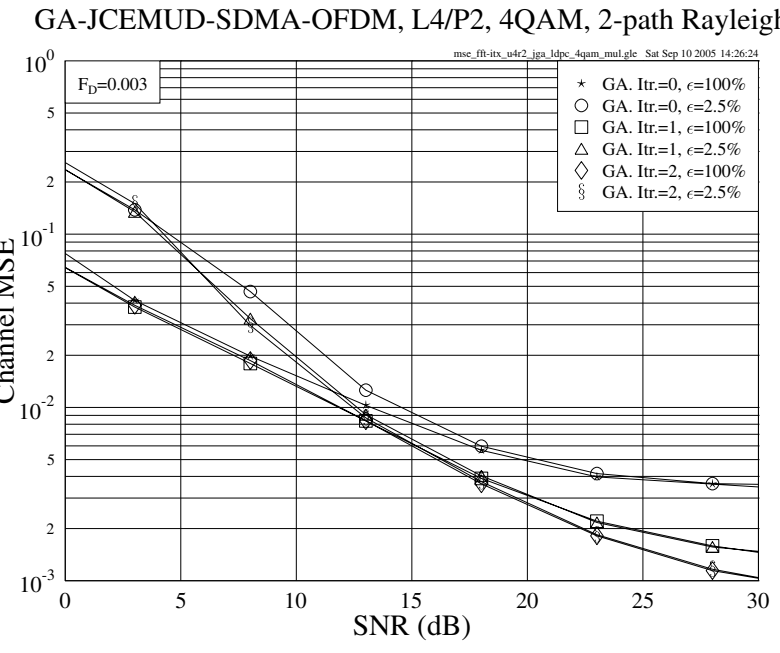

Fig. 5. Channel MSE versus SNR performance of the iterative GA-JCEMUD assisted SDMA-OFDM system in the rank-deficient scenario, where $L=4$ users were supported with the aid of $P=2$ receiver antenna elements, while invoking different numbers of iterations. The basic simulation parameters are given in Table I.

that the proposed GA-JCEMUD is capable of simultaneously capturing the fading envelope changes of each individual userreceiver link, regardless of its instant variety of fading. Since an equally good performance was attained over all the userreceiver links, this demonstrates the global robustness of the proposed approach in MIMO scenarios.

In Figure 7 the BER performance of the proposed system using different pilot overheads is investigated. In most cases, the GA-JCEMUD was capable of achieving a good performance using a pilot overhead as low as $\epsilon=1.5 \sim 2.5 \%$. Furthermore, the increase of pilot OFDM symbol overhead brings about more substantial benefits at the higher Doppler frequencies than at the lower ones, especially in the scenarios associated with higher SNRs, where an increasing fraction of the residual detection errors was inflicted by the inaccurate channel estimation.

In order to further characterize the advantages of the proposed GA-aided joint optimization scheme, in Figure 8 we compare the performances of the GA-JCEMUD and its counterpart, referred to as the GA-based channel estimator assisted OHRSA MUD (GACE-OHRSA-MUD), where the OHRSA MUD is serially concatenated with the stand-alone GA-aided channel estimator. More specifically, in the GACEOHRSA-MUD, channel estimation and symbol detection are separately accomplished by the GA-aided channel estimator and the OHRSA MUD, respectively. In other words, the symbol estimates offered by the OHRSA MUD are fixed during the GA-aided optimization process of the FD-CHTF estimates. Explicitly, in this case the effect of error propagation due to inaccurate symbol and/or channel estimates will become more severe, thus resulting in a dramatic BER performance degradation in comparison to the proposed joint optimization scheme, as evidenced in Figure 8. Furthermore, the superiority of the GA-JCEMUD becomes even more conspicuous in highDoppler scenarios.

Last but not least, we present the performance compari- 
True Channel Transfer Functions, 2-path Rayleigh

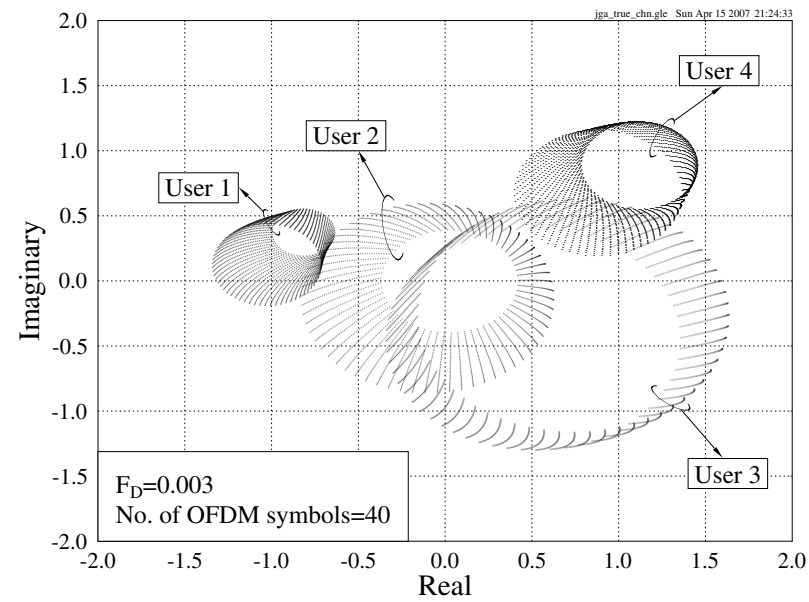

Estimated Channel Transfer Functions, 2-path Rayleigh

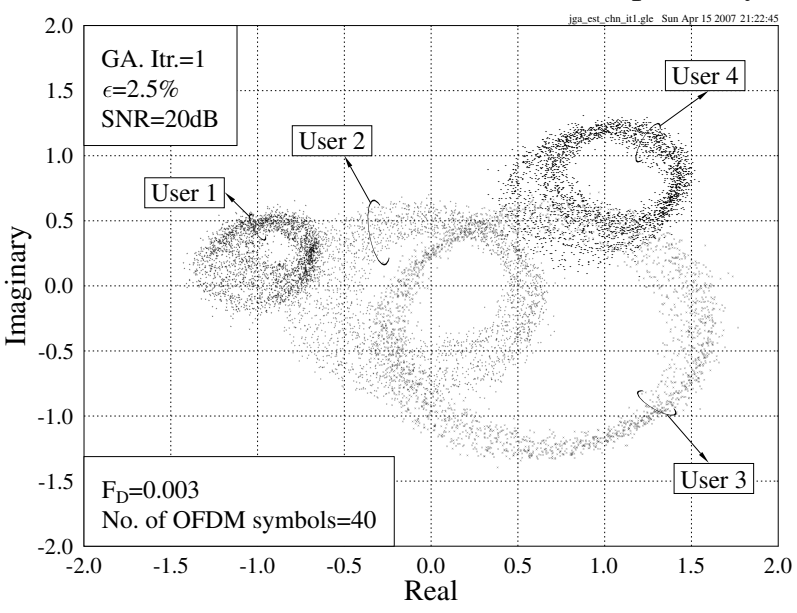

Fig. 6. Channel estimation performance of the iterative GA-JCEMUD assisted SDMA-OFDM system in the rank-deficient scenario, where $L=4$ users were supported with the aid of $P=2$ receiver antenna elements. The estimated FD-CHTFs $\hat{H}_{1}^{(l)}[n, k](l=1, \cdots, L ; k=$ $1, \cdots, K)$ associated with $n=1, \cdots, 40$ consecutive OFDM symbols at the $p=1^{\text {st }}$ receiver antenna are plotted at a SNR value of $20 \mathrm{~dB}$, and compared with the true FD-CHTFs. The basic simulation parameters are given in Table I.

son of the GA-JCEMUD providing either the conventional individual-based hard outputs [29], [32]-[35], [37], [38] or the proposed population-based soft outputs, as shown in Figure 9. As expected, with the advent of FEC codes, the proposed soft GA is capable of significantly outperforming the conventional arrangement, especially when the channel fades more rapidly. This result implies that the proposed GA exhibited a higher robustness against fast fading channels than the conventional GAs [29], [32]-[35], [37], [38].

It is also worth pointing out that the GA-based optimization imposes a significantly lower computational complexity than that of the optimum ML-based optimization, when perfect CSI is available. Typically, the Objective Function (OF) has to be evaluated for the entire search space by the ML MUD, whilst a substantially lower number of $\mathrm{OF}$ evaluations is required by the GA MUD. Comprehensive complexity comparisons between the ML- and GA-based MUDs can be found in [37],
LDPC-GA-JCEMUD-SDMA-OFDM, L4/P2, 4QAM, 2-path Rayleigh

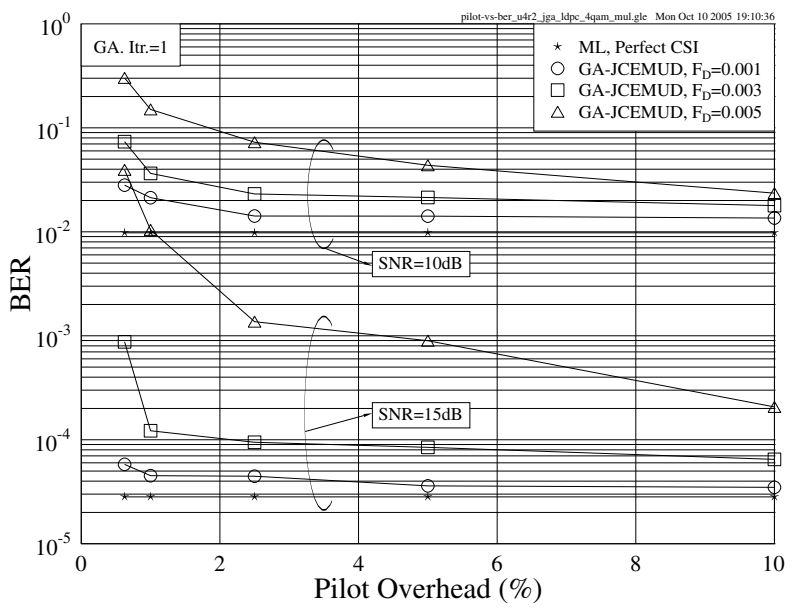

Fig. 7. BER versus pilot overhead performance of the LDPC-coded iterative GA-JCEMUD assisted SDMA-OFDM system in the rankdeficient scenario, where $L=4$ users were supported with the aid of $P=2$ receiver antenna elements, while assuming different values of the OFDM symbol normalized Doppler frequency $F_{D}$. The basic simulation parameters are given in Table I.

LDPC-GA-JCEMUD-SDMA-OFDM, L4/P2, 4QAM, 2-path Rayleigh

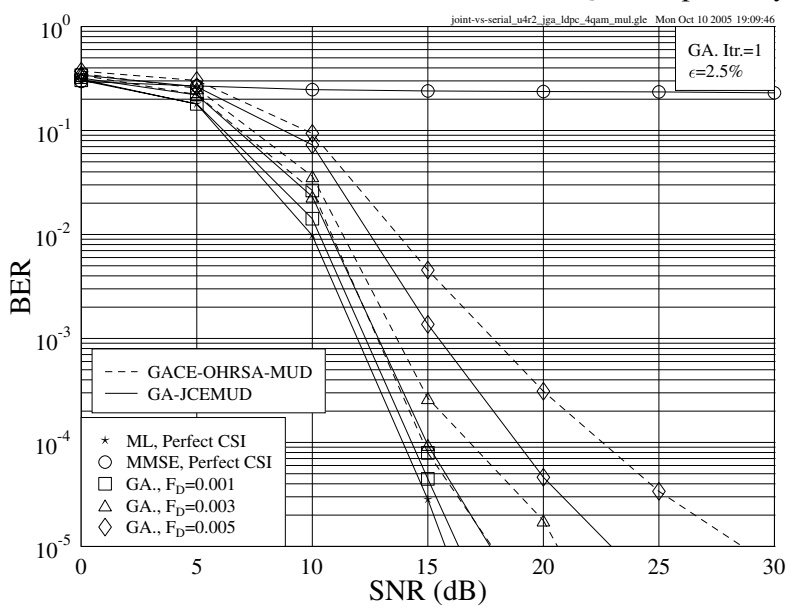

Fig. 8. Performance comparison of the LDPC-coded SDMAOFDM system using either the iterative GA-JCEMUD or the GACEOHRSA-MUD in the rank-deficient scenario, where $L=4$ users were supported with the aid of $P=2$ receiver antenna elements, while assuming different values of the OFDM symbol normalized Doppler frequency $F_{D}$. The basic simulation parameters are given in Table I.

[38]. On the other hand, when the CSI is imperfect, a higher GA population size and/or number of generations, as well as possibly a higher number of GA-JCEMUD iterations has to be used, in order to 'compensate' for the potential performance loss imposed by the imperfect channel knowledge. This is particularly true in rank-deficient scenarios, where the detrimental effect of imperfect CSI is further aggravated by the high MUI. In this challenging situation, the proposed GAbased joint optimization scheme still remains capable of maintaining a relatively lower complexity than that of the perfectCSI aided ML MUD, although the achievable complexity reduction is not as significant as that achieved by the GA 
LDPC-GA-JCEMUD-SDMA-OFDM, L4/P2, 4QAM, 2-path Rayleigh

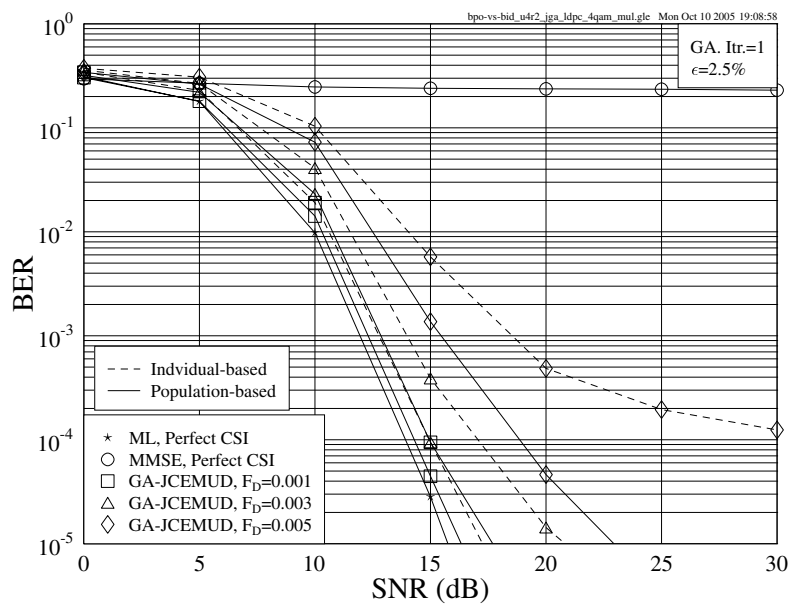

Fig. 9. Performance comparison of the LDPC-coded SDMA-OFDM system using either the conventional individual-based or the proposed population-based GA-JCEMUD in the rank-deficient scenario, where $L=4$ users were supported with the aid of $P=2$ receiver antenna elements, while assuming different values of the OFDM symbol normalized Doppler frequency $F_{D}$. The basic simulation parameters are given in Table I.

MUDs exploiting perfect channel knowledge. Nonetheless, the optimal parameter set for the GA-JCEMUD may be expected to further reduce the associated complexity, which requires further investigations.

\section{CONCLUSIONS}

From the investigations and discussions conducted in the previous sections, we conclude that the proposed GA-aided iterative joint channel estimation and multi-user detection scheme generating soft outputs constitutes an effective solution to the channel estimation problem in multi-user MIMO SDMA-OFDM systems. Furthermore, the GA-JCEMUD is capable of exhibiting a robust performance in rank-deficient scenarios, where the number of users is higher than the number of receiver antenna elements, either with or without FEC coding. This attractive property enables the SDMA-OFDM system to potentially support an increased number of users in comparison to that allowed by conventional techniques. Our future research will consider the design of similar down-link systems.

\section{REFERENCES}

[1] T. Keller and L. Hanzo, "Adaptive modulation techniques for duplex OFDM transmission," IEEE Trans. Veh. Technol., vol. 49, no. 5, pp. 1893-1906, Sept. 2000.

[2] — - "Adaptive multicarrier modulation: a convenient framework for time-frequency processing in wireless communications," Proc. of the IEEE, vol. 88, no. 5, pp. 611-640, May 2000

[3] B. J. Choi and L. Hanzo, "Optimum mode-switching-assisted constantpower single- and multicarrier adaptive modulation," IEEE Trans. Veh. Technol., vol. 52, no. 3, pp. 536-560, May 2003.

[4] L. Hanzo, M. Münster, B. J. Choi, and T. Keller, OFDM and MC-CDMA for Broadband Multi-user Communications, WLANs and Broadcasting. IEEE Press - John Wiley \& Sons Ltd., 2003.

[5] S. Verdu, Multiuser Detection. Cambridge University Press, 1998.

[6] M. Münster and L. Hanzo, "Parallel-interference-cancellation-assisted decision-directed channel estimation for OFDM systems using multiple transmit antennas," IEEE Trans. Wireless Commun., vol. 4, no. 5, pp. 2148-2162, Sept. 2005.
[7] H. Liu and G. Xu, "Smart antennas in wireless systems: uplink multiuser blind channel and sequence detection," IEEE Trans. Commun., vol. 45, no. 2, pp. 187-199, Feb. 1997.

[8] J. M. F. Xavier, V. A. N. Barroso, and J. M. F. Moura, "Closed-form blind channel identification and source separation in SDMA systems through correlative coding," IEEE J. Sel. Areas Commun., vol. 16, no. 8, pp. 1506-1517, Oct. 1998.

[9] J. Xavier, V. A. N. Barroso, and J. M. F. Moura, "Closed-form correlative coding (CFC2) blind identification of MIMO channels: isometry fitting to second order statistics," IEEE Trans. Signal Processing, vol. 49, no. 5, pp. 1073-1086, May 2001.

[10] I. Bradaric, A. P. Pertropulu, and K. I. Diamantaras, "Blind MIMO FIR channel identification based on second-order spectra correlations," IEEE Trans. Signal Processing, vol. 51, no. 6, pp. 1668-1674, June 2003.

[11] M. C. Necker and G. L. Stüber, "Totally blind channel estimation for OFDM on fast varying mobile radio channels," IEEE Trans. Wireless Commun., vol. 3, no. 5, pp. 1514-1525, Sept. 2004

[12] W. Nabhane and H. V. Poor, "Blind joint equalization and multiuser detection in dispersive MC-CDMA/MC-DS-CDMA/MT-CDMA channels," in Proc. 2002 IEEE Military Communications Conference (MILCOM '02), vol. 2, Oct. 2002, pp. 814-819.

[13] Y. Li, N. Seshadri, and S. Ariyavisitakul, "Channel estimation for OFDM systems with transmitter diversity in mobile wireless channels," IEEE J. Sel. Areas Commun., vol. 17, no. 3, pp. 461-471, March 1999.

[14] Y. Li, "Simplified channel estimation for OFDM systems with multiple transmit antennas," IEEE Trans. Wireless Commun., vol. 1, no. 1, pp. 67-75, Jan. 2002.

[15] H. Minn, D. I. Kim, and V. K. Bhargava, "A reduced complexity channel estimation for OFDM systems with transmit diversity in mobile wireless channels," IEEE Trans. Commun., vol. 50, no. 5, pp. 799-807, May 2002.

[16] Y. Li, J. H. Winters, and N. R. Sollenberger, "MIMO-OFDM for wireless communications: signal detection with enhanced channel estimation," IEEE Trans. Commun., vol. 50, no. 9, pp. 1471-1477, Sept. 2002.

[17] F. W. Vook and T. A. Thomas, "MMSE multi-user channel estimation for broadband wireless communications," in Proc. 2001 IEEE Global Telecommunications Conference (GLOBECOM '01), vol. 1, Nov. 2001, pp. $470-474$.

[18] S. Thoen, L. Deneire, L. V. D. Perre, M. Engels, and H. D. Man, "Constrained least squares detector for OFDM/SDMA-based wireless networks," IEEE Trans. Wireless Commun., vol. 2, no. 1, pp. 129-140, Jan. 2003.

[19] Y. Qiao, S. Yu, P. Su, and L. Zhang, "Research on an iterative algorithm of LS channel estimation in MIMO OFDM systems," IEEE Trans. Broadcast., vol. 51, no. 1, pp. 149-153, March 2005.

[20] Y. Zeng and T. S. Ng, "A semi-blind channel estimation method for multiuser multiantenna OFDM systems," IEEE Trans. Signal Processing, vol. 52, no. 5, pp. 1419-1429, May 2004.

[21] K. J. Kim and R. A. Iltis, "Joint detection and channel estimation algorithms for QS-CDMA signals over time-varying channels," IEEE Trans. Commun., vol. 50, no. 5, pp. 845-855, May 2002.

[22] K. J. Kim, J. Yue, R. A. Iltis, and J. D. Gibson, "A QRD-M/Kalman filter-based detection and channel estimation algorithm for MIMOOFDM systems," IEEE Trans. Wireless Commun., vol. 4, no. 2, pp. 710-721, March 2005.

[23] Z. Wang, Z. Han, and K. J. R. Liu, "A MIMO-OFDM channel estimation approach using time of arrivals," IEEE Trans. Wireless Commun., vol. 4, no. 3, pp. 1207-1213, May 2005 .

[24] M. Shin, H. Lee, and C. Lee, "Enhanced channel-estimation technique for MIMO-OFDM systems," IEEE Trans. Veh. Technol., vol. 53, no. 1, pp. 262-265, Jan. 2004.

[25] B. Lu and X. Wang, "Bayesian blind turbo receiver for coded OFDM systems with frequency offset and frequency-selective fading," IEEE $J$. Sel. Areas Commun., vol. 19, no. 12, pp. 2516-2527, Dec. 2001.

[26] Z. Yang and X. Wang, "A sequential Monte Carlo blind receiver for OFDM systems in frequency-selective fading channels," IEEE Trans. Signal Processing, vol. 50, no. 2, pp. 271-280, Feb. 2002.

[27] T. Cui and C. Tellambura, "Joint channel estimation and data detection for OFDM systems via sphere decoding," in Proc. 2004 IEEE Global Telecommunications Conference (GLOBECOM '04), vol. 6, pp. 36563660.

[28] H. Zhu, B. Farhang-Boroujeny, and C. Schlegel, "Pilot embedding for joint channel estimation and data detection in MIMO communication systems," IEEE Commun. Lett., vol. 7, no. 1, pp. 30-32, Jan. 2003.

[29] K. Yen and L. Hanzo, "Genetic algorithm assisted joint multiuser symbol detection and fading channel estimation for synchronous CDMA systems," IEEE J. Sel. Areas Commun., vol. 19, no. 6, pp. 985-998, June 2001 
[30] S. Chen and Y. Wu, "Maximum likelihood joint channel and data estimation using genetic algorithms," IEEE Trans. Signal Processing, vol. 46, no. 5, pp. 1469-1473, May 1998.

[31] C. E. Tan and I. J. Wassell, "Near-optimum training sequences for OFDM systems," in Proc. The 9th Asia-Pacific Conference on Communications (APCC '03), vol. 1, Sept. 2003, pp. 119-123.

[32] L. Hanzo, L.-L. Yang, E.-L. Kuan, and K. Yen, Single- and MultiCarrier DS-CDMA: Multi-User Detection, Space-Time Spreading, Synchronisation and Standards. IEEE Press - John Wiley \& Sons Ltd., 2003.

[33] C. Ergün and K. Hacioglu, "Multiuser detection using a genetic algorithm in CDMA communications systems," IEEE Trans. Commun., vol. 48, no. 8, pp. 1374-1383, Aug. 2000.

[34] K. Yen and L. Hanzo, "Antenna-diversity-assisted genetic-algorithmbased multiuser detection schemes for synchronous CDMA systems," IEEE Trans. Commun., vol. 51, no. 3, pp. 366-370, March 2003.

[35] — "Genetic-algorithm-assisted multiuser detection in asynchronous CDMA communications," IEEE Trans. Veh. Technol., vol. 53, no. 5, pp. 1413-1422, Sept. 2004

[36] X. Wu, T. C. Chuah, B. S. Sharif, and O. R. Hinton, "Adaptive robust detection for CDMA using a genetic algorithm," IEE Proc. Commun., vol. 150 , no. 6 , pp. 437-444, 10 Dec. 2003.

[37] M. Jiang and L. Hanzo, "Improved hybrid MMSE detection for turbo trellis coded modulation assisted multi-user OFDM systems," Electron. Lett., vol. 40, no. 16, pp. 1002-1003, Aug. 2004.

[38] M. Jiang, S. X. Ng, and L. Hanzo, "Hybrid iterative multi-user detection for channel coded space division multiple access OFDM systems," IEEE Trans. Veh. Techn., vol. 55, no. 1, pp. 115-127, Jan. 2006.

[39] J. Akhtman and L. Hanzo, "Reduced-complexity maximum-likelihood detection in multiple-antenna-aided multicarrier systems," in Proc. 5th International Workshop on Multi-Carrier Spread Spectrum Communications, Oberpfaffenhofen, Germany, Sept. 2005.

[40] — "An optimized-hierarchy-aided maximum likelihood detector for MIMO-OFDM," in Proc. 2006 IEEE 63rd Vehicular Technology Conference (VTC '06 Spring), vol. 3, May 2006, pp. 1526-1530.

[41] R. Gallager, "Low-density parity-check codes," IEEE Trans. Inf. Theory, vol. 8, no. 1, pp. 21-28, Jan. 1962.

[42] J. Akhtman and L. Hanzo, "Novel optimized-hierarchy RSA-aided space-time processing method," University of Southampton, Southampton, UK, Mobile VCE Core 3 Programme - Wireless Enablers 2.2: ICRWE2.2.1, May 2005.

[43] D. E. Goldberg, Genetic Algorithms in Search, Optimization, and Machine Learning. Reading, MA: Addison-Wesley, 1989.

[44] R. L. Haupt and S. E. Haupt, Practical Genetic Algorithms, 2nd ed. John Wiley \& Sons, Ltd., 2004.

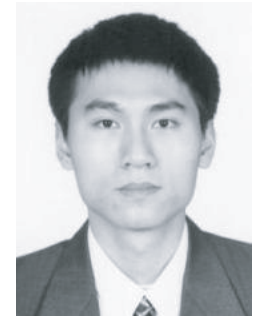

Ming Jiang (S'04-M'07) received B.Eng. and M.Eng. degrees in Electronics Engineering in 1999 and 2002 from South China University of Technology (SCUT), China, and Ph.D. degree in Telecommunications in 2006 from University of Southampton, UK, respectively. From 2002 to 2005, he was involved in the Core 3 research project of Mobile Virtual Centre of Excellence (VCE), UK on airinterface algorithms for MIMO OFDM systems. Since April 2006, Dr. Jiang has been with Advanced Technology, Standards and Regulation (ATSR) of Samsung Electronics Research Institute (SERI), UK, working on the European FP6 WINNER project as well as internal projects on advanced wireless communication systems. His research interests fall in the general area of wireless communications, including multi-user detection, channel estimation, spacetime processing, heuristic and adaptive optimization, frequency-hopping, MIMO OFDM and OFDMA systems, etc. Dr. Jiang has co-authored one IEEE Press book chapter, six IEE/IEEE journal papers, and eight IEE/IEEE conference papers.

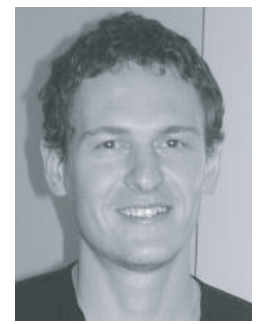

Jos Akhtman (M'00) received the B.Sc. degree in Physics and Mathematics in 2000 from the Hebrew University of Jerusalem, Israel. From 2000 to 2002 he was working as a Research Engineer in VYYO Ltd., Jerusalem, Israel. He is currently working towards the Ph.D. degree in the Communications Group, School of Electronics and Computer Science at the University of Southampton, UK. His major subject of interest is optimization algorithms for advanced multi-antenna multi-carrier communication systems. Specifically, iterative channel estimation, detection, space-time processing and turbo transceiver architecture.

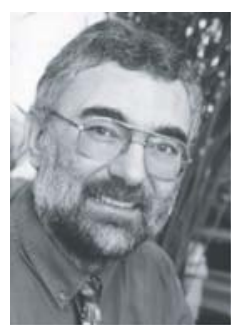

Lajos Hanzo (M'91-SM'92-F'04) Fellow of the Royal Academy of Engineering, received his firstclass degree in electronics in 1976 and his doctorate in 1983. In 2004 he was awarded the Doctor of Sciences (DSc) degree by the University of Southampton, UK. During his career in telecommunications he has held various research and academic posts in Hungary, Germany and the UK. Since 1986 he has been with the Department of Electronics and Computer Science, University of Southampton, UK, where he holds the chair in telecommunications. He has co-authored 15 books, totalling 10000 pages on mobile radio communications, published in excess of 700 research papers, has acted as TPC Chair of numerous major IEE and IEEE conferences, presented various keynote lectures and has been awarded a number of distinctions. Currently he heads an academic research team, working on a range of research projects in the field of wireless multimedia communications sponsored by industry, the Engineering and Physical Sciences Research Council (EPSRC) UK, the European IST Programme and the Mobile Virtual Centre of Excellence (VCE), $\mathrm{UK}$. He is an enthusiastic supporter of industrial and academic liaison and he offers a range of industrial courses. Lajos is also an IEEE Distinguished Lecturer of both the Communications as well as the Vehicular Technology Society, a Fellow of both the IEEE and the IEE. He is an editorial board member of the Proceedings of the IEEE and a Governor of the IEEE VT Society. For further information on research in progress and associated publications, please refer to http://www-mobile.ecs.soton.ac.uk 